\title{
ANÁLISE DE FÁRMACOS EM MATERIAL BIOLÓGICO: ACOPLAMENTO MICROEXTRAÇÃO EM FASE SÓLIDA “NO TUBO” E CROMATOGRAFIA LÍQUIDA DE ALTA EFICIÊNCIA
}

\author{
Maria Eugênia C. Queiroz* \\ Departamento de Química, Faculdade de Filosofia Ciências e Letras de Ribeirão Preto, Universidade de São Paulo, \\ Av. Bandeirantes, 3900, 14040-901 Ribeirão Preto - SP \\ Fernando M. Lanças \\ Instituto de Química de São Carlos, Universidade de São Paulo, CP 780, 13560-970 São Carlos - SP
}

Recebido em 13/7/04; aceito em 16/3/05; publicado na web em 30/6/05

\begin{abstract}
ANALYSIS OF DRUGS IN BIOLOGICAL SAMPLES: AUTOMATED “IN-TUBE” SOLID-PHASE MICROEXTRACTION AND HIGH PERFORMANCE LIQUID CHROMATOGRAPHY. A new solid phase microextraction (SPME) system, known as in-tube SPME, was recently developed using an open tubular fused-silica capilary column, instead of an SPME fiber, as the SPME device. On-line in-tube SPME is usually used in combination with high performance liquid chromatography. Drugs in biological samples are directly extracted and concentrated in the stationary phase of capillary columns by repeated draw/eject cycles of sample solution, and then directly transferred to the liquid chromatographic column. In-tube SPME is suitable for automation. Automated sample handling procedures not only shorten the total analysis time, but also usually provide better accuracy and precision relative to manual techniques. In-tube SPME has been demonstrated to be a very effective and highly sensitive technique to determine drugs in biological samples for various purposes such as therapeutic drug monitoring, clinical toxicology, bioavailability and pharmacokinetics.
\end{abstract}

Keywords: in-tube solid-phase microextraction; drugs; biological samples.

\section{INTRODUÇÃO}

A determinação de fármacos em amostras biológicas, principalmente no plasma de pacientes, coletadas com base no contexto clínico e nos princípios da farmacocinética, tem sido um procedimento usual na área clínica, para assegurar a eficácia terapêutica e minimizar os efeitos adversos (sintomas de toxicidade) dos fármacos prescritos.

O pré-tratamento de amostras biológicas, que abrange as etapas de extração, pré-concentração e purificação, tem sido requerido nas análises cromatográficas de fármacos, para eliminar interferentes (compostos endógenos ou outros fármacos administrados concomitantemente com os analitos) e aumentar a sensibilidade e seletividade analítica.

Os métodos convencionais, empregados em análises de rotina no tratamento de amostras biológicas, têm sido extração líquidolíquido e extração em fase sólida.

A técnica de extração líquido-líquido apresenta desvantagens, tais como, consumo de solventes orgânicos de alta pureza, exposição do analista a compostos tóxicos, várias etapas para sua execução e formação de emulsão entre as fases, o que resulta na perda do analito.

A extração em fase sólida (SPE) apresenta uma grande variedade de fases extratoras, resultando em diferentes tipos de interações com os analitos favorecendo, desta forma, a seletividade analítica; possibilita a automação das análises e o acoplamento em linha com técnicas cromatográficas. Entretanto, tem apresentado algumas limitações como o bloqueio dos poros da fase extratora pelos componentes da matriz, utilização de solventes orgânicos para a eluição, variações analíticas entre cartuchos extratores e várias etapas operacionais para sua execução.

*e-mail: mariaeqn@ffclrp.usp.br
A microextração em fase sólida (SPME), desenvolvida por Pawliszyn et $a l .{ }^{1}$ em 1990, apresenta uma série de vantagens em relação aos métodos convencionais de extração, ou seja, não requer instrumentação analítica sofisticada, rápido processo operacional, permite automação das análises, concentração dos analitos e reutilização das fibras extratoras. O processo de extração ou pré-concentração de analitos ocorre em micro escala. $\mathrm{O}$ dispositivo básico de SPME consiste de uma fibra de sílica fundida de $10 \mathrm{~mm}$ de comprimento e 110 a $160 \mu \mathrm{m}$ de diâmetro, recoberta com polímeros sorventes ou sólidos adsorventes, com espessuras de 7 a $100 \mu \mathrm{m}$.

A SPME baseia-se em um processo de equilíbrio entre fases. O sistema de extração consiste das fases aquosa (amostra homogênea), polimérica extratora (fibra) e gasosa. Durante a extração em um sistema trifásico considerado ideal, os analitos migram entre as três fases até que o equilíbrio de partição ou sorção seja atingido.

A extração tem sido realizada pela exposição da fibra diretamente na amostra (modo direto, Figura 1a), ou em sua fase gasosa. Após o processo SPME, os analitos sorvidos na fase polimérica da fibra têm sido determinados por diferentes técnicas analíticas. Em análises realizadas por cromatografia gasosa (SPME-GC), a fibra, após o processo de extração, é inserida no injetor aquecido e os analitos são rapidamente dessorvidos termicamente para a coluna cromatográfica; desta forma, as análises realizadas por SPME-GC não utilizam solventes orgânicos (Figura 1b) ${ }^{1,2}$.

A técnica SPME-GC, inicialmente desenvolvida para análise de poluentes em amostras de água ${ }^{3-6}$, tem sido empregada com êxito na extração de fármacos voláteis e semivoláteis em análises de amostras biológicas $^{7-32}$ (Tabela 1), principalmente quando extraídos da fase gasosa, no modo "headspace" $20,25,32$, onde não ocorre interação das proteínas da amostra biológica com a fibra. Já os fármacos menos voláteis ou termicamente instáveis têm sido ana-

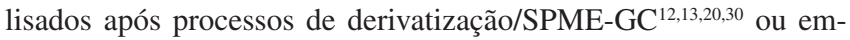




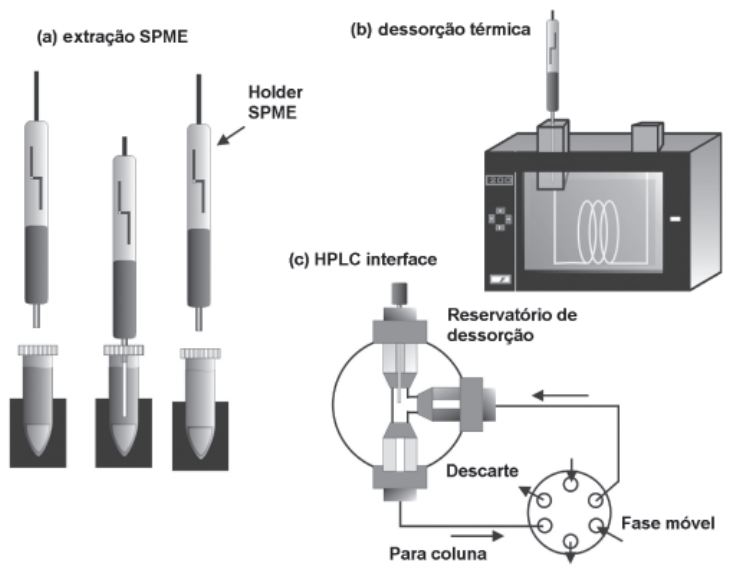

Figura 1. (a) processo de extração SPME; (b) processo de dessorção térmica $S P M E-G C$ e (c) processo de dessorção SPME-HPLC

pregando a técnica SPME acoplada à cromatografia líquida de alta eficiência (SPME-HPLC) $)^{33,34}$.

$\mathrm{O}$ acoplamento com SPME-HPLC ${ }^{33,34}$ requer uma interface apropriada, tal como a apresentada na Figura 1c, em forma de um $\mathrm{T}$, onde a fibra é inserida na extremidade superior e as demais extremidades, lateral e inferior, são conectadas à válvula de seis pórticos do HPLC. A dessorção dos analitos poderá ser realizada no modo dinâmico, com a fase móvel, ou, no modo estático, quando os analitos forem fortemente sorvidos na fibra. Neste último modo, a fibra, anterior à eluição dos analitos para a coluna cromatográfica, permanece na interface, em contato com um volume previamente estabelecido de fase móvel ou de solvente orgânico, durante um intervalo de tempo, para dessorção dos analitos.

As análises realizadas por SPME-HPLC apresentam algumas limitações, tais como dificuldades no acoplamento da interface/ HPLC, dessorção não eficiente dos compostos sorvidos pela fibra, análise manual, número limitado de fibras adequadas para análise de fármacos, principalmente os básicos e polares, e baixa estabilidade destas fibras, quando expostas à fase móvel ou a solventes orgânicos ${ }^{35}$.

Queiroz et al..$^{36}$ descreveram um sistema simples SPME-HPLC "off-line", o qual não requer a utilização de interface, para determinação simultânea de lamotrigina, carbazepina e 10, 11 epóxido carbamazepina em amostras de plasma.

\section{SPME "NO TUBO" ${ }^{37-47}$}

Um novo sistema SPME-HPLC, denominado SPME "no tubo", foi recentemente desenvolvido para microextração e pré-concentração de fármacos menos voláteis e/ou termicamente instáveis. Utiliza um tubo capilar aberto de sílica fundida (dispositivo SPME), com a superfície interna revestida com a fase estacionária (quimicamente ligada), o qual tem sido acoplado em linha com o sistema HPLC. Permite automação do processo de extração, resultando em maior precisão e menor tempo de análise, quando comparado às técnicas "off-line" convencionais. Como as amostras biológicas são injetadas no sistema praticamente em seu estado fisiológico, diminui a exposição dos analistas a estas amostras, além de minimizar perdas do analito durante o processo de extração.

O sistema SPME-HPLC "no tubo" pode ser montado fixandose uma coluna capilar entre a alça de amostragem e a agulha do injetor automático do HPLC. Como ilustra a Figura 2a, o injetor automático aspira a amostra do frasco e transporta-a para o capilar a uma velocidade linear constante. $\mathrm{O}$ volume de amostra aspirado é dispensado novamente no frasco do injetor automático. Estas etapas são referidas como ciclos aspirar/dispensar, os quais são executados repetitivamente segundo programas do injetor automático. Na coluna capilar ocorre o processo de partição ou sorção do analito com a fase estacionária, até que o equilíbrio seja atingido. $\mathrm{O}$ equilíbrio entre as fases tem sido atingido rapidamente, pois a extração é realizada sob agitação pelo fluxo da amostra, entrando e saindo da coluna capilar. Durante o processo de extração, a válvula do injetor HPLC deverá estar na posição carregar (Figura 2a). O capilar tem sido condicionado com metanol ${ }^{44}$ ou com a fase móvel ${ }^{37,40,42}$, antes do processo de extração.

Os analitos extraídos são dessorvidos rapidamente do revestimento do capilar pela vazão da fase móvel ou pela adição de um solvente orgânico, após posicionar a válvula do injetor HPLC na posição injetar (Figura 2b). Os analitos dessorvidos são transferidos para a coluna analítica do HPLC, para separação e detecção em ultravioleta (UV) ou espectrometria de massas (MS). Desta forma, a técnica SPME "no tubo" não necessita de uma interface especial SPME-HPLC para dessorção dos analitos.

Para evitar a presença de partículas no transporte das amostras biológicas ao longo do capilar, as proteínas têm sido removidas
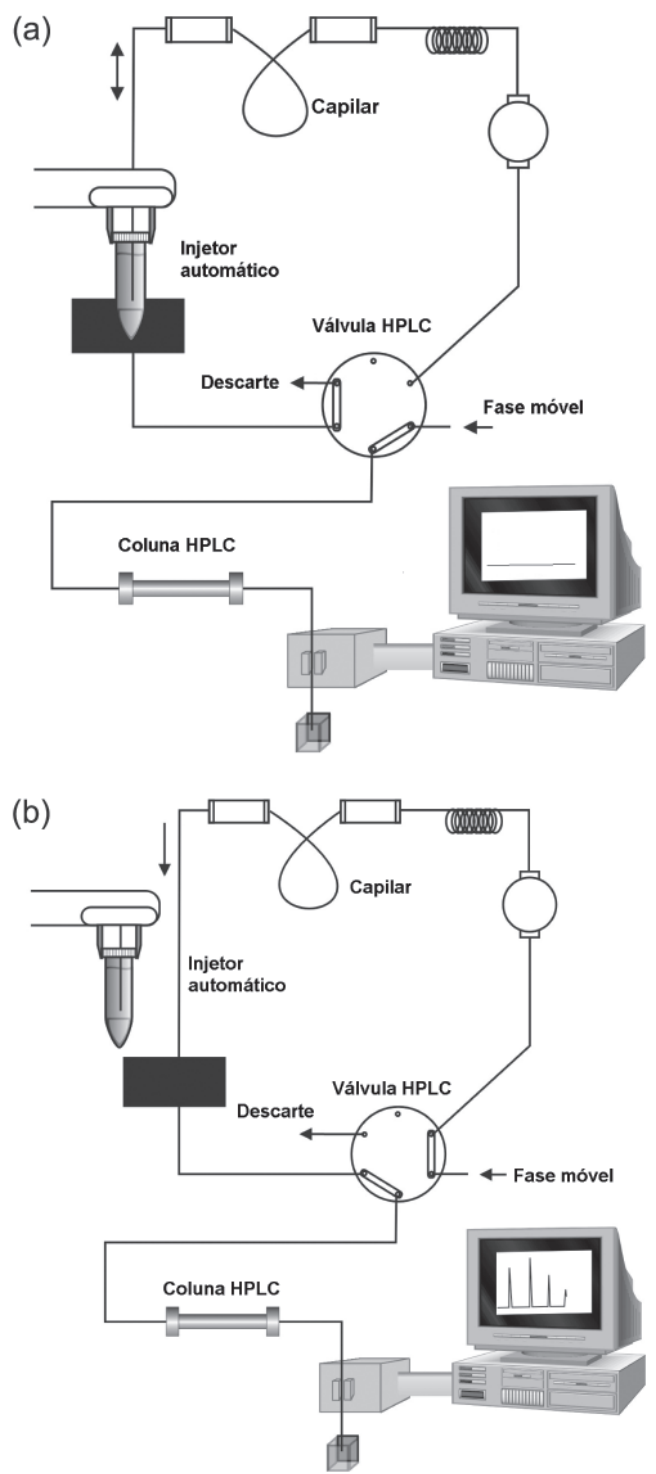

Figura 2. Sistema SPME-HPLC "no tubo": (a) extração, (b) dessorção 
Tabela 1. Aplicações da técnica SPME para análises de fármacos em amostras biológicas

\begin{tabular}{|c|c|c|c|c|}
\hline Fármacos(matriz) & $\begin{array}{l}\text { Modo de extração, } \\
\text { Fibra (espessura, } \mu \mathrm{m})\end{array}$ & Sistema analítico (LQ) & Observações & Ref. \\
\hline Ácido valpróico (plasma) & $\begin{array}{l}\text { Modo direto } \\
\text { PDMS (100) }\end{array}$ & $\begin{array}{c}\text { GC-FID } \\
\left(1 \mu \mathrm{g} \mathrm{mL}^{-1}\right)\end{array}$ & $\begin{array}{l}\text { Equilíbrio de diálise - } \\
\text { SPME }\end{array}$ & 7 \\
\hline $\begin{array}{l}\text { Anilina, fenóis, nitrobenzenos } \\
\text { (plasma) }\end{array}$ & $\begin{array}{l}\text { Modo direto } \\
\text { PA (85) }\end{array}$ & GC-MS & Estudo da ligação proteica & 8 \\
\hline Anestésicos (sangue) & $\begin{array}{l}\text { Modo direto } \\
\text { PDMS (100) }\end{array}$ & $\begin{array}{c}\text { GC-FID } \\
\left(54-158 \mathrm{ng} \mathrm{mL}^{-1}\right)\end{array}$ & $\begin{array}{c}\text { Extração após precipitação } \\
\text { de proteínas }\end{array}$ & 9 \\
\hline Antidepressivos (sangue) & $\begin{array}{l}\text { Modo direto } \\
\text { PDMS (100) }\end{array}$ & $\begin{array}{l}\text { GC-NPD, GC-MS } \\
\left(90-200 \mathrm{ng} \mathrm{mL}^{-1}\right)\end{array}$ & $\begin{array}{l}\text { Modelo teórico influência } \\
\text { das proteínas }\end{array}$ & 10 \\
\hline Antidepressivos (plasma) & $\begin{array}{l}\text { Modo direto } \\
\text { PDMS (100) }\end{array}$ & $\begin{array}{c}\text { GC-FID } \\
\left(16-25 \mathrm{ng} \mathrm{mL}^{-1}\right)\end{array}$ & Toxicologia forense & 11 \\
\hline Anfetaminas (urina) & $\begin{array}{l}\text { Modo direto } \\
\text { PDMS (100) }\end{array}$ & GC-NPD, GC-MS & $\begin{array}{c}\text { Derivatização da amostra } \\
\text { anterior SPME }\end{array}$ & 12 \\
\hline Anfetaminas (sangue) & $\begin{array}{l}\text { Modo direto } \\
\text { PDMS (100) }\end{array}$ & GC-MS & Derivatização no injetor & 13 \\
\hline Diazepam (plasma) & $\begin{array}{c}\text { Modo direto } \\
\text { PA }(85) \\
\text { PDMS }(7,100)\end{array}$ & $\begin{array}{c}\text { GC-FID } \\
\left(0,25 \mathrm{nmol} \mathrm{mL}^{-1}\right)\end{array}$ & $\begin{array}{c}\text { Fibra PA modificada com } \\
\text { 1-octanol, pré-tratamento } \\
\text { plasma (TCA) }\end{array}$ & 14 \\
\hline Benzodiazepínicos (plasma) & $\begin{array}{l}\text { Modo direto } \\
\text { PA (85) }\end{array}$ & $\begin{array}{c}\text { GC-FID } \\
\left(0,01-0,48 \mu \mathrm{mol} \mathrm{mL} \mathrm{mL}^{-1}\right)\end{array}$ & $\begin{array}{l}\text { Fibra PA modificada } \\
\text { com 1-octanol }\end{array}$ & 15 \\
\hline Clozapina (plasma) & $\begin{array}{l}\text { Modo direto } \\
\text { PDMS (100) }\end{array}$ & $\begin{array}{c}\text { GC-NPD } \\
\left(30 \mathrm{ng} \mathrm{mL}^{-1}\right)\end{array}$ & $\begin{array}{l}\text { Influência das proteínas } \\
\text { e triglicerídeos }\end{array}$ & 16 \\
\hline $\begin{array}{l}\text { Lidocaina e metabólitos } \\
\text { (plasma) }\end{array}$ & $\begin{array}{c}\text { Modo direto } \\
\text { CW-DVB (65) } \\
\text { PA (85) } \\
\text { PDMS (100) }\end{array}$ & $\begin{array}{c}\text { GC-NPD } \\
\left(8-21 \mathrm{ng} \mathrm{mL}^{-1}\right)\end{array}$ & Avaliação de diferentes fibras & 17 \\
\hline Lidocaina (plasma) & $\begin{array}{l}\text { Modo direto } \\
\text { PDMS (100) }\end{array}$ & $\begin{array}{c}\text { GC-FID } \\
\left(5 \mathrm{ng} \mathrm{mL}^{-1}\right)\end{array}$ & $\begin{array}{l}\text { Análise de lidocaina livre, } \\
\text { ligada à proteína e total }\end{array}$ & 18 \\
\hline Anestésicos (plasma) & $\begin{array}{l}\text { Modo direto } \\
\text { CW-DVB (65) } \\
\text { PA (85) }\end{array}$ & $\begin{array}{c}\text { GC-NPD } \\
\left(0.5 \mu \mathrm{mol} \mathrm{mL} \mathrm{mL}^{-1}\right)\end{array}$ & Ultrafiltração do plasma & 19 \\
\hline $\begin{array}{l}\text { Ácido gama-hidroxibutírico } \\
\text { (plasma) }\end{array}$ & $\begin{array}{l}\text { Fase gasosa } \\
\text { Derivatização } \\
\text { "headspace" }\end{array}$ & $\begin{array}{l}\text { GC/PICI-MS } \\
\left(1 \mu \mathrm{g} \mathrm{mL} \mathrm{mL}^{-1}\right)\end{array}$ & $\begin{array}{c}\text { Conversão gama-hidroxibutírico } \\
\text { a gama-butirolactona }\end{array}$ & 20 \\
\hline $\begin{array}{l}\text { Metadona e metabólitos } \\
\text { (plasma) }\end{array}$ & $\begin{array}{l}\text { Modo direto } \\
\text { PDMS (100) }\end{array}$ & $\begin{array}{c}\text { GC-MS } \\
\left(40 \mathrm{ng} \mathrm{mL}^{-1}\right)\end{array}$ & Análise de amostras de pacientes & 21 \\
\hline $\begin{array}{l}\text { Levomepromazina } \\
\text { (plasma) }\end{array}$ & $\begin{array}{l}\text { Modo direto } \\
\text { PDMS (100) }\end{array}$ & $\begin{array}{l}\text { GC-NPD } \\
\left(5 \mathrm{ng} \mathrm{mL}^{-1}\right)\end{array}$ & Monitorização terapêutica & 22 \\
\hline Midazolam (plasma) & $\begin{array}{l}\text { Modo direto } \\
\text { PA (85) }\end{array}$ & $\begin{array}{l}\text { GC-MS (SIM) } \\
\left(1,0 \mathrm{ng} \mathrm{mL}^{-1}\right)\end{array}$ & Monitorização terapêutica & 23 \\
\hline Anticonvulsivantes (plasma) & $\begin{array}{l}\text { Modo direto } \\
\text { CW-DVB (65) }\end{array}$ & $\begin{array}{c}\text { GC-TSD } \\
\left(0,05-0,2 \mu \mathrm{g} \mathrm{mL} \mathrm{m}^{-1}\right)\end{array}$ & Monitorização terapêutica & 24 \\
\hline Timol (plasma) & $\begin{array}{c}\text { Fase gasosa } \\
\text { "Headspace" } \\
\text { PDMS-DVB (65) }\end{array}$ & $\begin{array}{c}\text { GC-FDI } \\
\left(8,1 \mathrm{ng} \mathrm{mL}^{-1}\right)\end{array}$ & Clivagem enzimática & 25 \\
\hline Sulfentanil (plasma) & $\begin{array}{c}\text { Modo direto } \\
\text { PDMS-DVB (65) }\end{array}$ & $\begin{array}{c}\text { GC-MS } \\
\left(6,0 \mathrm{ng} \mathrm{mL}^{-1}\right)\end{array}$ & Influência do pH e força iônica & 26 \\
\hline Anfetaminas (cabelo e saliva) & $\begin{array}{l}\text { Modo direto } \\
\text { PDMS (100) }\end{array}$ & $\begin{array}{c}\text { GC-MS } \\
\left(0,7-1,9 \mathrm{ng} \mathrm{mL}^{-1}\right)\end{array}$ & Sem derivatização da amostra & 27 \\
\hline
\end{tabular}


Tabela 1. continuação

\begin{tabular}{|c|c|c|c|c|}
\hline Fármacos(matriz) & $\begin{array}{l}\text { Modo de extração, } \\
\text { Fibra (espessura, } \mu \mathrm{m})\end{array}$ & Sistema analítico (LQ) & Observações & Ref. \\
\hline Drogas de abuso (saliva) & $\begin{array}{l}\text { Modo direto e } \\
\text { Fase gasosa } \\
\text { PDMS (30) }\end{array}$ & $\begin{array}{c}\text { GC-MS } \\
\left(1-100 \mathrm{ng} \mathrm{mL}^{-1}\right)\end{array}$ & Toxicologia social & 28 \\
\hline Amitraz (plasma) & $\begin{array}{l}\text { Modo direto } \\
\text { PDMS (100) }\end{array}$ & $\begin{array}{c}\text { GC-TSD } \\
\left(20 \mathrm{ng} \mathrm{mL}^{-1}\right)\end{array}$ & Estudo de toxicidade em cães & 29 \\
\hline Busulfan (plasma) & $\begin{array}{l}\text { Modo direto } \\
\text { CW-DVB (65) }\end{array}$ & $\begin{array}{c}\text { GC-MS } \\
\left(20 \mathrm{ng} \mathrm{mL}^{-1}\right)\end{array}$ & Derivatização da amostra & 30 \\
\hline Barbitúricos (sangue, urina) & $\begin{array}{l}\text { Modo direto } \\
\text { PA (85) }\end{array}$ & $\begin{array}{l}\text { GC-MS } \\
\text { sangue }\left(0,05 \text { a } 1 \mu \mathrm{gL} \mathrm{m}^{-1}\right) \\
\text { urina }\left(0,01 \text { a } 0,6 \mu \mathrm{gL}^{-1}\right)\end{array}$ & $\begin{array}{l}\text { Toxicologia clínica } \\
\text { e forense }\end{array}$ & 31 \\
\hline Drogas de abuso (cabelo) & $\begin{array}{l}\text { Fase gasosa } \\
\text { PDMS (100) }\end{array}$ & $\begin{array}{c}\text { GC-MS } \\
(0,7 \mathrm{ng} / \mathrm{mg})\end{array}$ & "Screening" & 32 \\
\hline
\end{tabular}

LQ: limite de quantificação, TCA; ácido tricloroacético; PICI-MS: espectrometria de massas com ionização química; SIM: seleção do íon monitorado; PDMS: polidimetilsiloxano; PA: poliacrilato; PDMS-DVB: polidimetilsiloxano divinilbenzeno; CW-DVB: carbowax divinilbenzeno; NPD: detector de nitrogênio e fósforo; TSD: detector termiônico específico e FID: detector de ionização de chama.

através de filtragem em micro filtro com poros de 0,25-0,45 $\mu \mathrm{m}^{37}$, pela precipitação em micro concentrador a $10000 \mathrm{~g}$ por $20 \mathrm{~min}^{38} \mathrm{ou}$ pela diluição com solventes orgânicos ${ }^{39}$.

A eficiência da extração SPME tem sido determinada através da quantidade de analito extraída pela fase estacionária do capilar. Para as fases estacionárias que extraem os analitos baseados no processo de absorção, a quantidade de analito extraído pode ser expressa segundo a Equação 1

$\mathrm{n}_{\mathrm{A}}=\mathrm{K}_{\mathrm{A}} \mathrm{V}_{\mathrm{f}} \mathrm{V}_{\mathrm{s}} \mathrm{C}_{\mathrm{A}}^{\circ} /\left(\mathrm{K}_{\mathrm{A}} \mathrm{V}_{\mathrm{f}}+\mathrm{V}_{\mathrm{s}}\right)$

onde $n_{A}$ representa a quantidade de analito A extraído pela fase estacionária, quando estabelecido o equilíbrio de partição ou sorção entre as fases, $\mathrm{V}_{\mathrm{f}}$ e $\mathrm{V}_{\mathrm{s}}$ : os volumes da fase estacionária do capilar e da solução de amostra, respectivamente; $\mathrm{C}_{\mathrm{A}}^{\circ}$ : a concentração inicial de analito na amostra, e $\mathrm{K}_{\mathrm{A}}$ : o coeficiente de partição.

O tempo requerido para atingir o equilíbrio de partição ou sorção do analito entre as fases durante o processo SPME "no tubo" pode ser expresso segundo a Equação 2

$\mathrm{t}_{\mathrm{e}}=\mathrm{L}\left[1+\mathrm{K}_{\mathrm{A}} \mathrm{x}\left(\mathrm{V}_{\mathrm{f}} / \mathrm{V}_{\mathrm{v}}\right)\right] / \mu$

onde $\mathrm{L}$ representa o comprimento do capilar, $\mathrm{K}_{\mathrm{A}}$ : coeficiente de partição, $\mathrm{V}_{\mathrm{f}}$ : volume da fase estacionária do capilar, $\mathrm{V}_{\mathrm{v}}$ : volume livre do capilar, $\mu$ : velocidade linear da vazão do fluido biológico. Durante as extrações SPME as dimensões físicas: $L, V_{f}, V_{v}$ e o valor $\mathrm{K}_{\mathrm{A}}$ são fixos; desta forma, o tempo de extração depende da velocidade linear da vazão do fluido.

Para obtenção de eficiente processo SPME "no tubo"-HPLC, as variáveis: comprimento e fase estacionária do capilar, volume e ciclos de amostra aspirada/dispensada e velocidade linear da vazão, deverão ser otimizadas.

\section{Otimização do processo SPME "no tubo"}

\section{Seleção da coluna capilar}

Várias colunas capilares encontram-se disponíveis no comércio com diferentes fases estacionárias, diâmetros internos, comprimentos e espessuras de filme. A seleção da fase estacionária baseia-se na regra "similar solubiliza similar". Por ex.: em coluna de baixa polaridade como a fase líquida, polidimetilsilicone, compostos hidrofóbicos são retidos de forma seletiva; no entanto, compostos hidrofílicos apresentam maior afinidade com a fase polietileno glicol (maior polaridade).

O diâmetro interno, comprimento e espessura do filme do capilar estão linearmente relacionados com a quantidade de analitos extraídos; no entanto, o aumento das variáveis descritas poderá causar picos largos, com cauda e dessorção não quantitativa dos analitos. Na maioria das análises têm-se utilizado colunas capilares de 50 a $60 \mathrm{~cm}$ de comprimento, com finos filmes, as quais têm resultado em extrações eficientes e cromatogramas bem definidos, sem efeito de memória.

Embora colunas capilares com fase líquida quimicamente ligada (entrecuzada) sejam estáveis na presença de água ou solventes orgânicos, estas podem ser facilmente deterioradas por ácidos inorgânicos ou bases fortes. Desta forma, torna-se necessário avaliar a estabilidade da fase estacionária, quando exposta à fase móvel durante o processo de dessorção. Como exemplo, a coluna Omegawax $^{\circledR} 250$ foi utilizada em mais de 500 extrações sem alteração da eficiência nas extrações de anfetaminas ${ }^{38}$, antihipertensivos ${ }^{40}$ e ranitidina ${ }^{41}$.

Wu et al. ${ }^{37}$ desenvolveram um novo capilar de sílica fundida revestido com polipirrol (PPY). Este polímero, quando comparado às fases estacionárias utilizadas em capilares comerciais, apresentou extrações mais eficientes para as análises de anfetaminas em amostras de urina e cabelo ${ }^{37}, \beta$-bloqueadores na urina e soro $^{42} \mathrm{e}$ verapamil e principais metabólitos em várias matrizes biológicas, como plasma, urina e cultura de célula ${ }^{39}$. O capilar PPY mostrouse estável nas condições de análise, permitindo a utilização em centenas de determinações.

Para as análises de compostos benzodiazepínicos em amostras de urina e plasma foram utilizados os capilares Supel-Q PLOT ${ }^{\circledR}$ revestido com o polímero divinilbenzeno ${ }^{43}$, e RAM-ADS (acesso a material restrito - sílica quimicamente ligada a alquil diol) ${ }^{44}$, sendo que o último permitiu o fracionamento simultâneo das proteínas do soro e dos analitos hidrofóbicos.

Mullet et $a l .{ }^{45}$ desenvolveram um capilar utilizando um tubo PEEK (poliéter éter cetona) empacotado com partículas MIP 
("molecularly imprinted polymer") para análise de propanolol em amostras de soro.

\section{Efeito das modificações da amostra}

As fases estacionárias presentes nos capilares, em sua grande maioria, não são trocadoras de íons; extraem somente espécies quimicamente neutras presentes na amostra. Os ácidos fracos e bases poderão ser convertidos a sua forma neutra, na qual poderão ser extraídos. Para assegurar-se que $99 \%$ dos compostos ácidos, presentes em uma amostra, possam ser convertidos para a forma neutra, o $\mathrm{pH}$ deverá ter valor, no mínimo, duas unidades abaixo do pKa do analito. Para os analitos básicos, o $\mathrm{pH}$ deverá ter valor, no mínimo, duas unidades acima do $\mathrm{pKb}$ do analito.

Wu et al. ${ }^{42}$ obtiveram eficiente extração de $\beta$-bloqueadores, fármacos básicos, em $\mathrm{pH}$ 8,5 (Tampão tris- $\mathrm{HCl}, 100 \mathrm{mM}$ ) e em solução saturada com $\mathrm{NaCl}$.

$\mathrm{O}$ aumento da concentração de $\mathrm{NaCl}$ e do $\mathrm{pH}(3,0-10,0)$ da amostra resultou em extrações mais eficientes de anfetaminas ( $\mathrm{pKa}$ 9,5-10,0), quando se utilizou o capilar $\mathrm{PPY}^{37}$. O revestimento do capilar PPY e as anfetaminas (em baixos valores de $\mathrm{pH}$ ) encontram-se positivamente carregados, resultando em repulsão eletrostática entre analitos e revestimento da fibra. Já em altos valores de $\mathrm{pH}$ as cargas positivas dos analitos são reduzidas e interações intermoleculares são observadas entre PPY e analitos, aumentando, desta forma, a eficiência do processo SPME "no tubo" ${ }^{37}$. Para análise de verapamil e metabólitos ${ }^{39}$ (pKa 9,0 - 10,0), o pH 7,0 apresentou os melhores resultados.

Embora o efeito provocado pela adição de sais ("salting out") aumente a eficiência do processo SPME "no tubo", poderá causar obstrução do capilar através da deposição dos sais. A presença de solventes hidrofílicos na amostra diminui a eficiência da extração, pois aumenta a solubilidade dos analitos na mesma. A eficiência da extração não tem sido influenciada por adições de metanol em concentrações iguais ou menores a $5 \%$. Na análise de benzodiazepínicos em amostras de soro, $5 \%$ de metanol foi adicionado às amostras para liberação das proteínas ligadas aos fármacos ${ }^{45}$.

\section{Efeito do volume e número de ciclos de amostra aspiradal dispensada}

$\mathrm{O}$ volume e número de ciclos de amostra aspirada/dispensada estão linearmente relacionados com a capacidade do capilar e quantidade de analito extraído.

Uma coluna capilar de $60 \mathrm{~cm}$ de comprimento e $0,25 \mathrm{~mm}$ de diâmetro interno apresenta capacidade de $29,4 \mu \mathrm{L}$, já da agulha de injeção $(10 \mu \mathrm{L})$ à extremidade final da coluna, a capacidade resultante será de 39,4 $\mu \mathrm{L}$. O volume de amostra aspirada/dispensada tem sido de 30 a $40 \mu \mathrm{L}$ com velocidade linear de 50 a $100 \mu \mathrm{L} \mathrm{min}{ }^{-1}$. Abaixo desta velocidade, a extração requer longo tempo de análise e valores acima dos mencionados poderão ocasionar a formação de bolhas ao longo do capilar, reduzindo a eficiência da extração.

Em condição ideal, a amostra deverá ser aspirada/dispensada até atingir o equilíbrio de partição dos compostos com a fase estacionária. Este equilíbrio tem sido avaliado através de gráficos do rendimento da extração versus número de ciclos de amostra aspirada/injetada. No entanto, quando não é requerida alta sensibilidade analítica, extrações fora do equilíbrio têm sido realizadas com o objetivo de diminuir o tempo de análise.

A extração de verapamil e metabólitos ${ }^{39}$ foi realizada em capilar PPY com 40 ciclos de $30 \mu \mathrm{L}$ amostra e velocidade linear de $100 \mu \mathrm{L} \mathrm{m^{-1 }}$. O aumento da velocidade linear de 35 para $100 \mu \mathrm{L}$ $\min ^{-1}$ resultou em extrações mais eficientes em menor tempo de análise ${ }^{39}$. Mullett et $a l .{ }^{45}$ observaram que a diminuição da velocidade linear de 100 para $50 \mu \mathrm{L} \mathrm{min}{ }^{-1}$, durante 10 ciclos, resultou no aumento de eficiência do processo SPME para as análises de propanolol em amostras de soro. A diminuição da velocidade desta extração aumentou a transferência de massa do analito para os sítios de adsorção do capilar MIP.

Para extração de $\beta$-bloqueadores em amostras de urina e soro, 15 ciclos de $30 \mu \mathrm{L}$ de amostra foram transportados para o capilar PPY de $60 \mathrm{~cm}$ de comprimento. Aumento adicional no número de ciclos, no volume de amostra e no comprimento do capilar resultaram em maior tempo de análise e picos com cauda, sem aumento significativo na sensibilidade analítica ${ }^{42}$.

\section{Dessorção dos compostos do capilar}

A dessorção dos analitos do capilar tem sido rápida, pois a coluna capilar apresenta fase estacionária com espessura ao redor de $0,1 \mu \mathrm{m}$. Este processo tem sido realizado de dois modos: dinâmico (dessorção através da fase móvel) e estático (quando solvente orgânico é aspirado e transportado para o capilar). A dessorção estática tem sido aplicada para compostos fortemente adsorvidos à fase do capilar; neste caso, a dessorção deverá ser rápida e eficiente, com reduzido volume de solvente. Para um capilar (60 cm x $0,25 \mathrm{~mm}$ id) o processo de dessorção tem sido realizado aspirando $40 \mu \mathrm{L}$ de solvente. Deve-se considerar a capacidade da coluna e solubilidade do solvente com a fase móvel. Já para o modo dinâmico é possível a dessorção direta com a vazão da fase móvel, apenas girando a válvula do HPLC para a posição injetar.

Segundo os parâmetros de validação analítica, avaliados de acordo com normas regulamentadoras específicas da área clínica, a técnica SPME-HPLC "no tubo" tem sido aplicada com êxito a diferentes propósitos, tais como monitorização terapêutica, toxicologia clínica e estudos de farmacocinética ${ }^{37-47}$ (Tabela 2).

\section{CONCLUSÃO}

A técnica SPME-HPLC "no tubo" não requer uma interface específica para dessorção dos analitos, como a utilizada em SPME-HPLC convencional, permite a automação dos métodos cromatográficos, resultando em maior precisão e menor tempo de análise, quando comparado às técnicas manuais utilizadas em análises de rotina de fármacos em material biológico. Como as amostras biológicas são injetadas no sistema, praticamente em seu estado fisiológico, diminui a exposição dos analistas a estas amostras, além de minimizar perdas do analito durante o processo de extração, todo o analito extraído é introduzido no sistema analítico.

Para obtenção de eficiente processo SPME-HPLC "no tubo", as variáveis comprimento e fase estacionária do capilar, volume e ciclos de amostra aspirada/dispensada e velocidade linear da vazão deverão ser otimizadas durante o desenvolvimento de novas metodologias.

As principais vantagens da técnica SPME-HPLC "no tubo", quando comparada à SPE acoplada em linha com HPLC, têm sido simplicidade, rapidez, extração em micro escala, alta sensibilidade analítica, pequeno volume de amostra, baixo custo, reutilização do capilar extrator, fácil automação e reduzido volume de solvente orgânico. Tem sido aplicada com êxito na análise de compostos hidrofóbicos e hidrofílicos presentes em amostras biológicas, podendo ser facilmente acoplada a vários métodos analíticos, principalmente os em micro escala. 
Tabela 2. Aplicações da técnica "no tubo" SPME na análise de fármacos em amostras biológicas

\begin{tabular}{|c|c|c|c|c|}
\hline Fármacos (Matriz) & Ciclos, volume e vazão de amostra & Capilar & Detecção(LD) & Ref. \\
\hline Ranitidina (urina) & $\begin{array}{c}10 \text { ciclos de } 30 \mu \mathrm{L} \\
\text { amostra } \\
\text { Vazão: } 100 \mu \mathrm{L} \text { min }^{-1}\end{array}$ & $\begin{array}{l}\text { Omegawax } \\
250\end{array}$ & $\begin{array}{l}\text { HPLC-UV } \\
\left(1,4 \mathrm{ng} \mathrm{mL}^{-1}\right)\end{array}$ & 41 \\
\hline Antidepressivos (urina) & $\begin{array}{l}\text { Vazão:80 } \mu \mathrm{L} \min ^{-1} \\
\quad \mathrm{t}=10 \mathrm{~min}\end{array}$ & DB-1 & $\begin{array}{c}\mu \mathrm{LC}-\mathrm{UV} \\
\left(5 \mathrm{ng} \mathrm{mL}^{-1}\right)\end{array}$ & 46 \\
\hline$\beta$-bloqueadores (urina, soro) & $\begin{array}{c}15 \text { ciclos de } 30 \mu \mathrm{L} \\
\text { amostra } \\
\text { Vazão: } 100 \mu \mathrm{L} \min ^{-1}\end{array}$ & $\begin{array}{l}\text { Omegawax } \\
250\end{array}$ & $\begin{array}{c}\text { LC-MS } \\
\left(0,1-1,2 \mathrm{ng} \mathrm{mL} \mathrm{mL}^{-1}\right)\end{array}$ & 40 \\
\hline$\beta$-bloqueadores (urina, soro) & $\begin{array}{c}15 \text { ciclos de } 30 \mu \mathrm{L} \\
\text { amostra } \\
\text { Vazão: } 100 \mu \mathrm{L} \text { min }^{-1}\end{array}$ & $\begin{array}{l}\text { Polímero } \\
\text { PPY }\end{array}$ & $\begin{array}{c}\text { LC-MS } \\
\left(1,5-7,9 \mathrm{ng} \mathrm{mL}^{-1}\right)\end{array}$ & 42 \\
\hline Anfetaminas (urina) & $\begin{array}{c}15 \text { ciclos de } 35 \mu \mathrm{L} \\
\text { amostra } \\
\text { Vazão: } 100 \mu \mathrm{L} \text { min }^{-1}\end{array}$ & $\begin{array}{l}\text { Omegawax } \\
250\end{array}$ & $\begin{array}{c}\text { LC-MS } \\
\left(0,3-0,8 \mathrm{ng} \mathrm{mL} \mathrm{mL}^{-1}\right)\end{array}$ & 38 \\
\hline $\begin{array}{l}\text { Benzodiazepínicos } \\
\text { (soro, urina) }\end{array}$ & $\begin{array}{l}10 \text { ciclos de } 30 \mu \mathrm{L} \\
\text { amostra } \\
\text { Vazão: } 0,3 \mathrm{~mL} \mathrm{~min}{ }^{-1}\end{array}$ & $\begin{array}{l}\text { Supel-Q } \\
\text { PLOT }\end{array}$ & $\begin{array}{c}\text { LC-MS } \\
\left(0,02-2,0 \mathrm{ng} \mathrm{mL}^{-1}\right)\end{array}$ & 43 \\
\hline Propanolol (soro) & 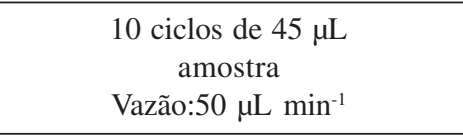 & MIP & $\begin{array}{l}\text { HPLC-UV } \\
\left(0,3 \mu \mathrm{g} \mathrm{mL} \mathrm{mL}^{-1}\right)\end{array}$ & 45 \\
\hline Anfetaminas (urina, cabelo) & $\begin{array}{c}10 \text { ciclos de } 40 \mu \mathrm{L} \\
\text { amostra } \\
\text { Vazão: } 100 \mu \mathrm{L} \text { min }^{-1}\end{array}$ & $\begin{array}{l}\text { Polímero } \\
\text { PPY }\end{array}$ & $\begin{array}{c}\text { LC-MS } \\
\left(8-56 \mathrm{ng} \mathrm{L}^{-1}\right)\end{array}$ & 37 \\
\hline Antidepressivos (urina) & Vazão:80 $\mu \mathrm{L}$ min $^{-1}$ & HM / DB-5 & CE-UV(44 - 153 ng mL-1) & 47 \\
\hline $\begin{array}{l}\text { Benzodiazepínicos } \\
\text { (soro) }\end{array}$ & $\begin{array}{c}4 \text { ciclos de } 20 \mu \mathrm{L} \\
\text { amostra } \\
\text { Vazão: } 20 \mu \mathrm{L} \min ^{-1}\end{array}$ & RAM / ADS & $\begin{array}{c}\text { HPLC-UV } \\
\left(24-26 \mathrm{ng} \mathrm{mL}^{-1}\right)\end{array}$ & 44 \\
\hline $\begin{array}{l}\text { Verapamil e metabólitos } \\
\text { (plasma, urina) }\end{array}$ & $\begin{array}{c}40 \text { ciclos de } 30 \mu \mathrm{L} \\
\text { amostra } \\
\text { Vazão: } 100 \mu \mathrm{L} \mathrm{min}{ }^{-1}\end{array}$ & $\begin{array}{l}\text { Polímero } \\
\text { PPY }\end{array}$ & $\begin{array}{c}\text { LC-MS } \\
\left(5-8 \mathrm{ng} \mathrm{mL}^{-1}\right)\end{array}$ & 39 \\
\hline
\end{tabular}

CE: eletroforese capilar; PPY: polipirrol; MIP: “molecularly imprinted polymer"; HM: Zilon fibra "high modulus"; RAM/ADS: material de restrito acesso/silica quimicamente ligada alquil diol; $\beta$-bloqueadores: nadolol, pindolol, acebutolol, timolol, metoprolol, oxprenolol, labetalol, propanolol, alprenolol. Benzodiazepínicos: clonazepam, axazepam, temazapem, nordazepam, diazepam. Antidepressivos: desipramina, nortriptina, imipramina e amitriptilina.

\section{REFERÊNCIAS}

1. Em Applications of solid phase microextraction; Pawliszyn, J., ed.; Royal Society of Chemistry: Cambridge, 1999.

2. Pawliszyn, J.; J. Chromatogr. Sci. 2000, 38, 270.

3. Queiroz, M. E. C.; Silva, S. M.; Carvalho, D.; Lanças, F. M.; J. Environ. Sci. Health 2001, 36, 517.

4. Lanças, F. M.; Souza, D.; J. Environ. Sci. Health 2003, 38, 417.

5. Lanças, F. M.; Rodrigues, R.; Lacerda, C. A.; J. Chromatogr. Sci. 2002, 40, 489.

6. Lanças, F. M.; Barrionuevo, W. R.; J. Environ. Sci. Health 2002, 69, 123.

7. Krogh, M.; Johansen, K.; Rasmussen, K. E.; J. Chromatogr., B: Biomed. Sci. Appl. 1995, 673, 299.

8. Vaes, W. H. J.; Ramos, E. U.; Verhaar, H. J. M.; Seinen, W.; Hermens, J. L. M.; Anal. Chem. 1996, 68, 4458.

9. Kumazawa, T.; Sato, K.; Seno, H.; Suzuki, O.; Chromatographia 1996, 43, 59.

10. Ulrich, S.; Martens, J.; J. Chromatogr., B: Biomed. Sci. Appl. 1997, 696, 217.

11. Lee, X. P.; Kumazawa, T.; Sato, K.; Suzuki, O.; J. Chromatogr. Sci. 1997, 35,302 .

12. Ugland, H. G.; Krogh, M.; Rasmussen, K. E.; J. Pharm. Biomed. Anal. 1999, 19, 463 .
13. Nagasawa, N; Yashiki, M.; Iwasaki, Y.; Kojima, T.; Forensic Sci. Int. 1996, $78,95$.

14. Krogh, M.; Grefslie, H.; Rasmussen, K. E.; J. Chromatogr., B: Biomed. Sci. Appl. 1997, 689, 357.

15. Reubsaet, K. J.; Norli, H. R.; Hemmersbach, P.; Rasmussen, K. E.; J. Pharm. Biomed. Anal. 1998, 18, 667.

16. Ulrich, S.; Kruggel, S.; Weigmann, H.; Hiemke, C.; J. Chromatogr., B: Biomed. Sci. Appl. 1999, 731, 231.

17. Abdel-Rehim, M.; Bielenstein, M.; Arvidsson, T.; J. Microcolumn Sep. 2000, 12, 308

18. Koster, E. H. M.; Wemes, C.; Morsink, J. B.; Jong, G. J.; J. Chromatogr. B: Biomed Sci. Appl. 2000, 739, 175.

19. Abdel-Rehim, M.; Carlsson, G.; Bielenstein, M.; Arvidsson, T.; Blomberg. L. G.; J. Chromatogr. Sci. 2000, 38, 458.

20. Frison, G.; Tedeschi, L.; Maietti, S.; Ferrara. S. D.; Rapid Commun. Mass Spectrom. 2000, 14, 2401

21. Bermejo, A. M.; Seara, R.; Tebernero, M. J.; Fernandez, P.; Marsili, R.; J. Anal. Toxicol. 2000, 24, 66.

22. Kruggel, S.; Ulrich. S.; Ther. Drug Monit. 2000, 22, 723.

23. Frison, G.; Tedeschi, L.; Maietti, S.; Ferrara. S. D.; Rapid Commun. Mass Spectrom. 2001, 15, 2497.

24. Queiroz, M. E. C.; Silva, S. M.; Carvalho, D.; Lanças, F. M.; J. Chromatogr. Sci. 2002, 40, 219. 
25. Kohlert, C.; Abel, G.; Schmid, E.; Veit. M.; J. Chromatogr., B: Biomed. Sci. Appl. 2002, 767, 11.

26. Paradis, C.; Dufresne, C.; Bolon, M.; Boulieu. R.; Ther. Drug Monit. 2002, 24, 768.

27. Gentili, S.; Torresi, A.; Marsili, R.; Chiarotti, M.; Marcchia T.; J. Chromatogr., B: Biomed. Sci. Appl. 2002, 780, 183.

28. Fucci, N.; De Giovanni, N.; Chiarotti, M.; Forensic Sci. Int. 2003, 134, 40.

29. Queiroz, M. E. C.; Valadão, C. A. A.; Farias, A.; Carvalho, D.; Lanças, F. M.; J. Chromatogr., B: Biomed. Sci. Appl. 2003, 794, 337.

30. Abdel-Rehim, M.; Hassan, Z.; Blomberg, L.; Hassan, M.; Ther. Drug Monit. 2003, 25, 400 .

31. Iwai, M.; Hattori, H.; Arinobu, T.; Noguchi, H.; Seno, H.; J. Chromatogr., B: Biomed. Sci. Appl. 2004, 806, 65.

32. Gentili, S.; Cornetta, M.; Macchia, T.; J. Chromatogr., B: Biomed. Sci. Appl. 2004, 801, 289.

33. Walles, M.; Mullett, W. M.; Pawliszyn, J.; J. Chromatogr., A 2004, 1025, 85.

34. Jinno, K.; Taniguchi, M.; Hayashida, M.; J. Pharm. Biomed. Anal. 1998, 17. 1081 .
35. Queiroz, M. E. C.; Lanças, F. M.; LCGC 2004, 22, 970.

36. Queiroz, M. E. C.; Silva, S. M.; Carvalho, D.; Lanças. F. M.; J. Sep. Sci. 2002, 25, 91 .

37. Wu, J.; Lord, H.; Pawliszyn, J.; Talanta 2001, 54, 655.

38. Kataoka, H.; Lord, H. L.; Kataoka, H.; J. Anal. Toxicol. 2000, $24,257$.

39. Walles, M.; Mullet, W. M.; Levsen, K.; Borlak, J.; Wunsch, G.; Pawliszyn, J.; J. Pharm. Biomed. Anal. 2002, 30, 307.

40. Kataoka, H.; Lord, H.; Yamamoto, S.; Narimatsu, S.; Pawliszyn, J.; J. Microcolumn Sep. 2000, 12, 493.

41. Kataoka, H.; Lord, H.; Pawliszyn, J.; J. Chromatogr., B: Biomed. Sci. Appl. 1999, 731, 353

42. Wu, J.; Lord, H.; Pawliszyn, J.; Kataoka, H.; J. Microcolumn Sep. 2000 , 12, 255.

43. Yuan, H.; Mester, Z.; Lord, H.; Pawliszyn, J.; J. Anal. Toxicol. 2000, 24, 718

44. Mullett, W. M.; Levsen, K.; Lubda, D.; Pawliszyn, J.; J. Chromatogr., A 2002, 963, 325

45. Mullett, W. M.; Martin, P.; Pawliszyn, J.; Anal. Chem. 2001, 73, 2383.

46. Saito, Y.; Kawazoe, M.; Hayashida, M.; Jinno, K.; Analyst 2000, 125, 807.

47. Jinno, K.; Kawazoe, M.; Saito, Y.; Takeichi, T.; Hayashida, M.; Eletrophoresis 2001, 22, 3785. 